\title{
Coronavirus Disease-2019: An Overview and Update
}

\author{
Agnibha Maiti ${ }^{1}$, Anup K Bhattacharya ${ }^{2}$ \\ Bengal Physician Journal (2019): 10.5005/jp-journals-10070-6126
}

There is current worldwide outbreak of a new type of coronavirus (COVID-19), which has originated from Wuhan, China, and has now spread to 140 other countries including India. As per recent available data, it can be said that spread to human has occurred via transmission from the wild animals sold in Wuhan seafood market. It spread rapidly through China affecting more than 85,000 people. Within a few months, it engulfed Europe causing massive loss of lives and property in Italy, Spain, France, Germany, the United Kingdom, and then the United States. It is now gaining a foothold in India, which has the second largest population in the world. As per current data, 4,429,969 people have been infected and 3 lakhs have succumbed to the illness all over the world. The WHO announced that COVID-19 has become a global health problem on March 11, 2020. Before March 3, India had three cases of coronavirus in Kerala, all of which were treated and discharged. On March 3, India's fourth case was diagnosed in the state of Rajasthan and it was later found that this patient had infected 17 other Italians who were on a tour to India. Till the date of writing this article, India has 78,003 confirmed cases and 2,549 deaths have taken place. Maharashtra, Delhi, and Tamil Nadu are the three most badly affected states.

Coronaviruses comprise a big family of viruses, which is responsible for illness to humans and animals. In humans, coronaviruses can cause different types of respiratory infections ranging from mild cough and cold to severe diseases such as severe acute respiratory syndrome (SARS) (2003) and Middle East respiratory syndrome (MERS) (2012). Newly discovered coronavirus is causing COVID-19. The virus that is responsible for COVID-19 and the virus that was the causative agent of SARS in 2003 are similar to each other intrinsically. But the illnesses they cause are completely nonidentical. SARS was most fatal but less contagious than COVID-19. Since 2003, nowhere in the world SARS caused any epidemic. Understanding the transmission risk is incomplete. One person gets infected from another person mainly via respiratory droplets. When infected person coughs, sneezes, or speaks, the virus is released in respiratory secretion and can infect another person. Infection may occur if a person makes contact with a contaminated surface (e.g., table, ticket counter, door handle, handrail of staircase, elevators) and then touches his/her eyes, nose, or mouth. Droplets hopefully do not go beyond more than 6 feet. ${ }^{1}$ Symptoms of COVID-19 typically manifest 4-5 days after exposure, although the incubation period is 14 days. The range of symptomatic infection varies from mild to severe. Most infections are not critical. Few common clinical findings are fever, dry cough, and tiredness. ${ }^{2,3}$ Some patients may present with headache, bodyache, nasal congestion, anosmia, sore throat, or diarrhea. These symptoms are mild in most of the cases and start very slowly. Some people even with active infection do not develop any symptom and also do not feel any uneasiness. Most people recover from the disease even without any treatment. Apart from conventional features, COVID-19 may present with some uncommon clinical features: neurological (COVID encephalopathy
${ }^{1}$ Department of Medicine, Institute of Postgraduate Medical Education and Research, Seth Sukhlal Karnani Memorial Hospital, Kolkata, West Bengal, India

${ }^{2}$ Department of Medicine, Agartala Government Medical College, Agartala, Tripura, India

Corresponding Author: Agnibha Maiti, Department of Medicine, Institute of Postgraduate Medical Education and Research, Seth Sukhlal Karnani Memorial Hospital, Kolkata, West Bengal, India, Phone: +919330952581, e-mail: agnivam@gmail.com

How to cite this article: Maiti A, Bhattacharya AK. Coronavirus Disease-2019: An Overview and Update. Bengal Physician Journal 2019;6(3):45-47.

Source of support: Nil

Conflict of interest: None

that manifests as acute necrotizing encephalopathy with brain scan showing abnormal swelling and inflammation at several regions, presentation as $G B$ syndrome also noted in a few case series, acroparesthesia, i.e., tingling and numbness of extremities may also occur), cardiac (myocarditis with elevated cardiac enzymes with reduced ejection fraction but without coronary artery disease), dermatological (COVID toes, i.e., young patients with purple-like lesions at toes due to localized inflammation or microclots, urticarial wheels are also reported in some patients, diffuse maculopapular eruption is also seen in few patients), ophthalmology (conjunctivitis/red eye). About $14 \%$ patients may present with severe disease that may manifest as dyspnea (RR $\geq$ 30 ), hypoxia (blood oxygen saturation $<94 \%$ ), lung infiltrate $>50 \%$ of the lung field within $24-48$ hours, $\mathrm{PaO}_{2} / \mathrm{FiO}_{2}$ ratio $<300$ in ABG. Only $5 \%$ of patients may manifest with critical disease, i.e., ARDS with respiratory failure, septic shock, and sepsis with multiorgan dysfunction. The overall case fatality rate is $2.5-5 \%$. The older age group and patients who have other comorbidities like diabetes, hypertension, chronic kidney disease, liver disease, and chronic obstructive pulmonary disease are more prone to infection and have a high case fatality rate. ${ }^{4}$ In published case series, males have been affected more than females overall. ${ }^{5}$ In patients with COVID-19 infection, WBC count is often variable. Various types of laboratory finding like leukopenia, leukocytosis, and lymphopenia have been described in various studies. Elevated lactate dehydrogenase, ferritin, procalcitonin, and elevated aminotransferase level have also been described. Most common finding in chest CT of a COVID19 patient is bilateral ground glass opacification with or without consolidative abnormalities. The patient with suspicion should undergo collection of nasopharyngeal or oropharyngeal swab and detection of SARS-CoV-2 RNA by reverse transcription polymerase chain reaction. IgG and IgM antibodies against SARS-CoV-2 can be detected by immunochromatography. This detection of antibodies is done in human whole blood, serum, and plasma. 
At present, no specific therapy is available to treat the infection, so prevention and reducing transmission in the society is the best way to combat the infection. Home management is recommended for patients with mild infections. Home isolation is needed for $>72$ hours afebrile or 7 days after symptom onset, whichever is longer or two negative samples 24 hours apart. Moderate to severe cases should be admitted and treated with oxygen supplementation, antipyretics, antitussive, antibiotics (azithromycin + amoxiclav is preferred) as indicated. Hydroxychloroquine (HCQS) 400 mg bd for 1 day followed by 200 $\mathrm{mg}$ bd for 5 days may be considered. The mechanism of action of HCQS includes inhibition of viral enzymes or processes such as viral DNA and RNA polymerase, viral protein glycosylation, virus assembly, new virus particle transport, and viral release. Other mechanism may also involve ACE2 cellular receptor inhibition, acidification at the surface cell membrane inhibiting fusion of virus, and immunomodulation of cytokine release. A number of antiviral agents are being surveyed for treatment of COVID-19. Remdesivir is a broad-spectrum antiviral classified as a nucleotide analog that has an activity against SARS-CoV-2. ${ }^{6,7}$ It acts as an inhibitor of RNA-dependent RNA polymerases. By this mechanism, it causes premature termination of viral RNA transcription. Adult dose is $200 \mathrm{mg}$ intravenous to be given as loading dose on 1st day followed by $100 \mathrm{mg}$ intravenous in the next 9 days. Lopinavirritonavir, combined protease inhibitor, which is primarily used for HIV infection, has in vitro activity against SARS-CoV-2. Do not combine hydroxychloroquine with lopinavir in view of drug interaction. In China, this combination is used in conjunction with interferon alfa for treatment of some patients with COVID-19 but does not reveal significant decrease in time to improvement or in mortality at 28 days. ${ }^{8-10}$ Immunomodulators are also being studied for stoppage of cytokine release, which is probably one of the most important reasons for development of SARS and shock in COVID-19 patients (e.g., tocilizumab and sarilumab are both monoclonal antibodies against IL- 6 receptor). ${ }^{11,12}$ The WHO and CDC recommend not to use glucocorticoids in patients with COVID-19 pneumonia unless there are other indications (e.g., acute exacerbation of COPD). As ACE-2 is thought to be a receptor for SARS-CoV-2, the patient receiving ACE inhibitor has an increased risk of adverse outcome with COVID-19 infection but there is no strong evidence to discontinue this medication. Antibodies to SARS-CoV-2 are present in the plasma of those who have recovered from the disease. In addition to clinical trials, a licensed physician can collect convalescent plasma from an individual patient through process of single-patient Emergency Investigational New Drug (EIND) applications. Patients eligible under EIND: (1) must have COVID-19 confirmed by laboratory testing, (2) must have severe or immediate life-threatening COVID-19, (3) RR > 30/ minute, (4) $\mathrm{SPO}_{2} \leq 93 \%$, (5) $\mathrm{PaO}_{2}: \mathrm{FiO}_{2}<300$, (6) septic shock, (7) MODS, and (8) within 24-48 hours lung infiltration more than $50 \%$. Hydroxychloroquine may have some role in prevention of COVID-19. Asymptomatic healthcare workers and asymptomatic household contact of laboratory-confirmed cases should take $400 \mathrm{mg}$ twice a day on day 1, followed by $400 \mathrm{mg}$ once weekly for next 7 weeks - to be taken with meals. Altimmune's intranasal coronavirus vaccine, INO-4800 by Inovio Pharmaceuticals, mRNA1273 vaccine by Moderna, Avian Coronavirus Infectious Bronchitis Virus (IBV) vaccine by MIGAL, and many more vaccines for SARSCoV-2 are in the developing stage only.
Following are the some necessary steps for avoiding community spread:

- Meticulous handwashing, especially needed after touching any surfaces. Hand sanitizer with at least $60 \%$ alcohol is a good option to clean the hands.

- Maintenance of respiratory hygiene is crucial (e.g., covering the cough or sneeze).

- The person who has respiratory symptom should use a triplelayer disposable surgical mask.

- Avoid crowded places. Close contact with individuals who are ill should also be avoided. A safe distance of $1 \mathrm{~m}$ should also be maintained.

- Shaking of hands and kissing should be avoided.

- Gatherings and nonessential travels should also be avoided.

- Pens may be used for switching on or off lights in common areas and also in lift buttons.

- At hospitals, avoid keeping patients files on bed.

- Use gloves while cleaning and disinfecting objects and surfaces that are frequently touched.

- We should be very careful when dealing with used mask and other personal protective equipment. These items are potential source of infection and should be discarded separately in a waste disposable bag.

It is normal to feel sad, stressed, confused, scared, or angry during a crisis; contacting people whom you trust may be helpful. During your homestay maintain a healthy lifestyle, which should include: nutritious diet, sleep, exercise, and talking to people whom you like. Smoking, alcohol, or taking sedative drugs must not be chosen as an alternative to tackle your emotion. If you feel overburdened, help of a health worker or counselor may be taken. Like any epidemic corona cannot be prevented without social bonding or initiative. Social distancing excludes it. Therefore, we must talk about physical distancing and social bonding (PDSB) instead of social distancing while fighting corona. The poor, underprivileged, and elderly can be supported by this social bonding. Health is a right and a social responsibility. Social distancing denies it but physical distancing and social bonding takes up that task.

\section{References}

1. Van Dreamland N, Bushmaker T, Morris DH, et al. Aerosol and surface stability of SARS-CoV-2 as compared with SARS-CoV-1. N Engl J Med 2020;382(16):1564-1567. DOI: 10.1056/NEJMc2004973.

2. Huang C, Wang $Y$, Li X, et al. Clinical features of patients infected with the 2019 novel coronavirus in Wuhan, China. Lancet 2020;395(10223):497-506. DOI: 10.1016/S0140-6736(20)30183-5.

3. Chen N, Zhou M, Dong X, et al. Epidemiological and clinical characteristics of 99 cases of 2019 novel coronavirus pneumonia in Wuhan, China: a descriptive study. Lancet 2020;395(10223):507-513. DOI: 10.1016/S0140-6736(20)30211-7.

4. CDC COVID-19 Response Team. Severe outcomes among patients with coronavirus disease 2019(COVID-19) - United States, February 12-March 16, 2020. MMWR Morb Mortal Wkly Rep 2020;69(12): 343-346. DOI: 10.15585/mmwr.mm6912e2.

5. Chan JFW, Yuan S, Kok K-H, et al. A familial cluster of pneumonia associated with 2019 novel coronavirus indicating person to person transmission: a study of a family cluster. Lancet 2020;395(10223):514523. DOI: 10.1016/S0140-6736(20)30154-9.

6. Sheahan TP, Sims AC, Leist SR, et al. Comparative therapeutic efficacy of remdesivir and combination lopinavir, ritonavir, and interferon beta against MERS-CoV. Nat Commun 2020;11(1):222. DOI: 10.1038/ s41467-019-13940-6. 
7. Agostini ML, Andres EL, Sims AC, et al. Coronavirus susceptibility to the antiviral remdesivir (GS-5734) is mediated by the viral polymerase and the proof reading exoribonuclease. mBio 2018;9(2):e00221. DOI: 10.1128/mBio.00221-18.

8. Harrison C. Coronavirus puts drug repurposing on the fast track. Nat Biotechnol 2020;38(4):379-381. DOI: 10.1038/d41587-020-00003-1.

9. Jin $\mathrm{YH}$, Cai L, Cheng Z-S, et al. A rapid advice guideline for the diagnosis and treatment of 2019 novel coronavirus (2019-nCoV) infected pneumonia (standard version). Mil Med Res 2020;7(1):4. DOI: 10.1186/s40779-020-0233-6.
10. Cao $B$, Wang $Y$, Wen $D$, et al. A trial of lopinavir-ritonavir in adults hospitalized with severe Covid-19. N Engl J Med 2020;382(19):17871799. DOI: 10.1056/NEJMoa2001282.

11. Alhazzani W, Møller MH, Arabi YM, et al. Surviving sepsis campaign: guidelines on the management of critically ill adults with coronavirus disease 2019(COVID-19). Crit Care Med 2020;48(6):e440-e469. DOI: 10.1097/CCM.0000000000004363.

12. Clinical Pharmacology powered by Clinical Key: Sarilumab. Clinical Key website. Accessed April16, 2020. https://www. clinicalkey.com/\#!/ content/drug-monograph/6-s2.0-4949. 